\title{
A New Numerical Simulation Method for Three-Dimensional Mesoscale Analysis of Concretes
}

\author{
Laizhong Song, ${ }^{1}$ Biwei Song, ${ }^{2}$ Tao Shen, ${ }^{1}$ and Bo Yu ${ }^{1}$ \\ ${ }^{1}$ College of Science, China Three Gorges University, Yichang, Hubei 443002, China \\ ${ }^{2}$ School of Mathematics and Statistics, Wuhan University, Wuhan 430072, China \\ Correspondence should be addressed to Bo Yu; yubo2003@amss.ac.cn
}

Received 3 August 2014; Accepted 19 December 2014

Academic Editor: Oleg V. Gendelman

Copyright (c) 2015 Laizhong Song et al. This is an open access article distributed under the Creative Commons Attribution License, which permits unrestricted use, distribution, and reproduction in any medium, provided the original work is properly cited.

\begin{abstract}
This paper presents a method for generating a region with a lot of randomly distributed ellipsoids. Using the parametric expression of an ellipsoid, the criterion for determining if a spatial point is in the interior or exterior of the ellipsoid is established. Then the computation of the distance between a point and the ellipsoid is converted into finding the solution to an optimization problem, which can be efficiently approximated by the searching method. Based on these facts, the proposed method is able to make the distance between generated ellipsoids very small and then successfully improve the content of ellipsoids grains in the region. Numerical results show that the proposed method can generate simulation of specimens in which the content of ellipsoids is higher than 55\% according to four-graded aggregates, $50 \%$ according to three-graded aggregates, and $45 \%$ according to two-graded aggregates, respectively, in relatively short time.
\end{abstract}

\section{Introduction}

Concrete is an important construction material because of its general availability and versatility. According to [1], there are four levels of the study of concretes: macrolevel, mesolevel, microlevel, and nanolevel. Mesolevel analysis of concrete is most popular because it can be used for further analysis of important properties of concrete such as deformation and damage resistance [2], mechanical properties [3], and electrical, thermal, and elastic properties [4]. At mesolevel, the concrete is treated as consisting of reinforced aggregates, mortar contents, and, optionally, interfacial zones between the aggregates and the mortar content, where the reinforced aggregates play the most important role.

For the mesolevel study of concrete, it is first necessary to generate a random aggregate structure in which the shape, size, and distribution of the reinforced aggregates resemble real concrete in the statistical sense. For this purpose, a lot of researchers contributed from different aspects. For example, in $[5,6]$, the authors proposed a procedure for generating random aggregates structures for rounded and angular aggregates based on the Monte Carlo random sampling principle and developed a method of mesh generation using the advancing front approach to do the nonlinear finite element analysis. In $[7,8]$, the authors tried to understand the aggregates structure of concrete from the viewpoint of mathematics. In [9], the authors developed a stochasticheuristic algorithm for generating the composite structure of concrete in 3-D space. The authors of [10] discussed in detail how to accurately model the mesoscale geometry with efficient numerical analysis of high resolution, where the ellipsoids were chosen to simulate the reinforced aggregates. In [11] the authors presented an effective method to generate the random distribution domains with large numbers of grains using the compactness algorithm and selection algorithm. In [12], the authors proposed a mesoscale model of concrete where the reinforced particles were generated according to a prescribed grading curve and placed randomly into the specimen. In [13], the authors presented a generating method based on an approximate solution of a quadratic programming with quadratic constraints. Comparing all these methods, it is difficult to say which one is the best because each one has advantages in some aspects and disadvantages in some other aspects. Furthermore, all the existing methods suffer from the same disadvantage, that is, low volume ratio of aggregates in the whole specimen. To generate a more realistic 
specimen which has a high content of reinforced aggregates, we need to develop a more efficient algorithm, which is the main purpose of this paper.

In this paper we propose a new method with which a simulated specimen of concrete, according to four-graded aggregates, can be generated where the content of aggregates can be higher than 55\%. The basic idea is to take advantage of the parametric expression of ellipsoid, with which we can determine if a spatial point is in the interior or exterior of the ellipsoid. Moreover, the distance between a point and the ellipsoid is converted into finding the solution to an optimization problem, which can be efficiently approximated by the searching method. Because of these facts, the proposed method is able to make the distance between ellipsoids very small and then successfully improve the content of ellipsoids grains in the region. The foundation of the algorithm will be described in Section 2. The algorithm itself will be presented in Section 3. Section 4 will be devoted to giving numerical results and corresponding discussion. The conclusion will be drawn in Section 5.

\section{Foundation of the Algorithm}

In this section we will describe the parametric equation of an ellipsoid, from which the criterion to find the relation between a spatial point and the ellipsoid is derived. Then we will explain how to approximately calculate the distance between the spatial point and the ellipsoid. The error analysis is given in the end of this section.

We assume an ellipsoid is centered at $O_{0}\left(x_{0}, y_{0}, z_{0}\right)$ with semiprincipal axes of lengths $a, b, c$, where $a \geq b \geq c>0$. The nutation angle, precession angle, and orbit-spin angle of the ellipsoid are designated as $\theta, \psi$, and $\varphi$, respectively. Set a matrix $A=\left(a_{i j}\right), i, j=1,2,3$, where

$$
\begin{gathered}
a_{11}=\cos \varphi \cos \psi-\sin \varphi \cos \theta \sin \psi ; \\
a_{12}=\cos \varphi \sin \psi+\sin \psi \cos \theta \cos \varphi ; \\
a_{13}=\sin \varphi \sin \theta ; \\
a_{21}=-\sin \varphi \cos \psi-\cos \varphi \cos \theta \sin \psi ; \\
a_{22}=\cos \varphi \cos \theta \cos \psi-\sin \varphi \sin \psi ; \\
a_{23}=\cos \varphi \sin \theta ; \quad a_{31}=\sin \theta \sin \psi ; \\
a_{32}=-\sin \theta \cos \psi ; \quad a_{33}=\cos \theta
\end{gathered}
$$

The domains of $\theta, \psi$, and $\varphi$ are $0 \leq \theta<\pi, 0 \leq \psi<2 \pi$, and $0 \leq \varphi<2 \pi$, respectively. Now the surface of the ellipsoid can be parameterized as

$$
S:\left(\begin{array}{l}
x \\
y \\
z
\end{array}\right)=A\left(\begin{array}{c}
a \cos u \sin v \\
b \sin u \sin v \\
c \cos v
\end{array}\right)+\left(\begin{array}{l}
x_{0} \\
y_{0} \\
z_{0}
\end{array}\right),
$$

where $(u, v) \in[0,2 \pi) \times[0, \pi]$. If we set $B=$ $\operatorname{diag}\left(1 / a^{2}, 1 / b^{2}, 1 / c^{2}\right)$ and $=\left(x-x_{0}, y-y_{0}, z-z_{0}\right)$, then the generalized equation of this ellipsoid is given as

$$
S: X A B A^{\prime} B^{\prime} X^{\prime}=1 \text {. }
$$

Now we define a function $f$ at $(x, y, z) \in \mathbb{R}^{3}$ by

$$
f(x, y, z)=X A B A^{\prime} X^{\prime}-1
$$

then we have the following proposition.

Proposition 1. For any $P \in \mathbb{R}^{3}$, if $f(P)>0$ then $P$ is in the exterior of the ellipsoid; if $f(P)=0$ then $P$ is on the surface of the ellipsoid; if $f(P)<0$ then $P$ is in the interior of the ellipsoid.

Now we are ready to consider the distance from an arbitrary spatial point $Q\left(x_{1}, y_{1}, z_{1}\right)$ to the surface of an ellipsoid defined in (2) or (3). That is, we define the function $F$ at $(x, y, z) \in \mathbb{R}^{3}$ by $F(x, y, z)=\left(x-x_{1}\right)^{2}+\left(y-y_{1}\right)^{2}+\left(z-z_{1}\right)^{2}$ and consider the following quantity:

$$
\begin{aligned}
& d(Q, S)=(\min \{F(x, y, z): \\
& \left.\left.\quad f(x, y, z)=0,(x, y, z) \in \mathbb{R}^{3}\right\}\right)^{1 / 2} .
\end{aligned}
$$

Of course this quantity equals

$$
\begin{aligned}
& d(Q, S)=(\min \{F(x, y, z): \\
& \left.\left.\quad(x, y, z) \in \mathbb{R}^{3} \text { satisfies }(2)\right\}\right)^{1 / 2} .
\end{aligned}
$$

Theoretically, there are analytical methods for solving the extremal problems defined in (5) and (6). The Lagrange multiplier can be directly used to solve (5). The extremal problem in (6) can be converted into an unconstraint minimum problem with respect to a function in variables $u$ and $v$. However, both methods need to solve a system of nonlinear equations, which is extremely inefficient in numerical calculation. Therefore, we are going to use a searching method to get an approximation solution to the extremal problem (6) in the following algorithm.

Algorithm 2 (computing the distance between a spatial point and an ellipsoid).

Step 1. Given a natural number $n$, take $u_{i+1}=i \pi n / 2, v_{i+1}=$ $i \pi / n, i=0, \ldots, n-1$.

Step 2. Calculate $n^{2}$ points on the surface of the ellipsoid $S$ according to (2); store them by $P_{i j}\left(x\left(u_{i}, v_{j}\right), y\left(u_{i}\right.\right.$, $\left.\left.v_{j}\right), z\left(u_{i}, v_{j}\right)\right), i, j=0,1, \ldots, n-1$.

Step 3. Calculate the function values of $F$ at these $n^{2}$ points; store them by $F_{i j}=F\left(x\left(u_{i}, v_{j}\right), y\left(u_{i}, v_{j}\right), z\left(u_{i}, v_{j}\right)\right), i, j=$ $0,1, \ldots, n-1$.

Step 4. Take $d(P, S) \approx \min \left\{F_{i j}, i, j=0, \ldots, n-1\right\}$ as the desired approximation to the actual distance.

Before we present the algorithm for the simulation of a region with a lot of ellipsoids as aggregates, we end this section by giving an error analysis of Algorithm 2. Since ellipsoids are closed and convex surfaces, for any point $P$ outside of an ellipsoid, there is one point $\bar{P}$ on the surface $S$ of the ellipsoid such that $d(P, S)=d(P, \bar{P})=\overline{P \bar{P}}$, where we 


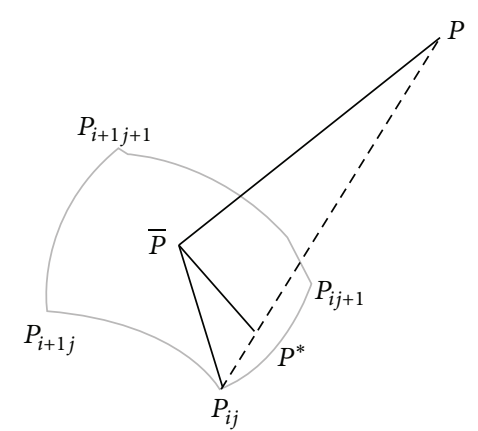

FIGURE 1: Error analysis.

use $\overline{A B}$ to denote the distance between point $A$ and point $B$. By the geometric characterization of the best approximation, $P \bar{P}$ is on the normal line of $S$ at the point $\bar{P}$. As shown in Figure 1 , let us set $P^{*}$ as the intersection point of $P P_{i j}$ and the arc which is centered at $P$ and the length of radius is $\bar{P} \bar{P}$. Without loss of generality, we can assume $d\left(P, P_{i j}\right)$ to be the approximation to $d(P, \bar{P})$. Noticing that $\overline{P \bar{P}}=\overline{P P^{*}}$, the error between the approximation $\overline{P P_{i j}}$ and the actual distance $\overline{P \bar{P}}$ is $\overline{P_{i j} P^{*}}$. It is clear that $\overline{P_{i j} P^{*}} \leq \overline{P_{i j} \bar{P}}+\overline{P^{*} \bar{P}}$. When $n$ is sufficiently large, $\overline{P^{*} \bar{P}} \leq \overline{P_{i j} \bar{P}}$, therefore, $\overline{P_{i j} P^{*}} \leq 2 \overline{P_{i j} \bar{P}}$. Thus, we have $0 \leq \overline{P_{i j} P^{*}} \leq 2 \overline{P_{i j} \bar{P}} \leq 2 \overline{P_{i j} P_{i+1 j+1}}$. By invoking the fact that $\overline{P_{i j} P_{i+1 j+1}}=\sqrt{\left(\overline{P_{i j} P_{i+1 j}}\right)^{2}+\left(\overline{P_{i j} P_{i j+1}}\right)^{2}}, \overline{P_{i j} P_{i j+1}}<\widehat{P_{i j} P_{i j+1}} \leq$ $2 a \pi / n$, and $\overline{P_{i j} P_{i+1 j}}<\widehat{P_{i j} P_{i+1}} \leq 2 a \pi / n$, where $\widehat{P_{i j} P_{i j+1}}$ and $\widehat{P_{i j} P_{i+1}}$ denote the length of the arc whose radius is $a$, we conclude

$$
0 \leq \overline{P_{i j} P^{*}} \leq \frac{4 \sqrt{2} a \pi}{n}
$$

In practical computation, (7) can be used to control the computational accuracy.

\section{Algorithm for Simulating the Domain with a Large Number of Randomly Distributed Ellipsoid Grains}

In the engineering computation, the computer simulation of the concrete always contains the aggregates agreeing with some probability distribution, which is obtained by the statistics for the components and shapes of the materials to be simulated. Based on this fact, we assume, without loss of generality, the following two statements.

(1) The simulated region is a cuboid.

(2) All the parameters including the ellipsoids center $\left(x_{0}, y_{0}, z_{0}\right)$, the semiprincipal axes lengths $a, b, c$, the nutation angle $\theta$, precession angle $\psi$, and orbit-spin angle $\varphi$ of the ellipsoids satisfy a uniform distribution. Of course we can modify the parameters such that they satisfy some other kind of distribution. Under these assumptions, we can develop a fast algorithm for generating a rectangular domain with a large amount of randomly distributed ellipsoids. The basic idea is as follows.

(a) According to the generated ellipsoids, we first randomly generate a point, such as $P$, in the simulation area. Then we determine whether it is in the exterior of all the generated ellipsoids. If so, we calculate the distances from the point $P$ to all the generated ellipsoids and find the minimum distance.

(b) If the minimum distance is bigger than the biggest semiprincipal axes length $a$ of the ellipsoid to be generated, then we generate a new ellipsoid with the biggest semiprincipal axle length $a$ and the center at $P$.

To describe the algorithm in detail, we use $l$ to denote the number of generated desired ellipsoids and $L$ the ellipsoids' total number, $v$ to denote the volume of the generated desired ellipsoids and $V$ the ellipsoids' total volume, and $k$ to denote the number of ellipsoids which are continuously rejected and $K$ the total number of rejected star domains.

Algorithm 3 (generating a cuboid with a lot of randomly distributed ellipsoids).

Step 1. Input all the parameters for generating the randomly distributed ellipsoids as well as the prescribed value of $L, V$, and $K$. Set $l=1, k=0$, and $v=0$.

Step 2. Randomly generate a number $a$ which can be a candidate for the biggest semiprincipal axes length of the desired ellipsoid.

Step 3. Randomly generate a point $\left(x_{0}, y_{0}\right)$ in the simulation domain and determine whether it is in the exterior of all the generated ellipsoids. If not, set $k=k+1$ and go to Step 4; otherwise, calculate the distances between $\left(x_{0}, y_{0}\right)$ and all the generated ellipsoids using Algorithm 2 and determine whether the minimum of the distances $d$ is bigger than or equal to $a$. If not, set $k=k+1$ and go to Step 4; otherwise, generate an ellipsoid with the center of $O\left(x_{0}, y_{0}, z_{0}\right)$, the biggest semiprincipal axle length $a$, and the other randomly chosen parameters such as the middle semiprincipal axle length $b(\leq a)$, the smallest semiprincipal axle length $c(\leq b)$, the nutation angle $\theta$, precession angle $\psi$, and orbit-spin angle $\varphi$. Calculate its volume $v_{0}$, and set $v=v+v_{0}, l=l+1$; go to Step 4.

Step 4. If $l>L$, or $k>K$, or $v>V$, end; otherwise, go to Step 2.

In practical computation, there are some techniques to improve the computational speed. For example, when we calculate the smallest distance between $O\left(x_{0}, y_{0}, z_{0}\right)$ and all the generated ellipsoids if $O\left(x_{0}, y_{0}, z_{0}\right)$ is in the exterior of them, we can implement it by the idea of approximation. That is, we calculate the distance $d^{\prime}$ between $O\left(x_{0}, y_{0}, z_{0}\right)$ and all the centers of the generated ellipsoids. If $d^{\prime}$ is bigger than 
the sum of $a$ and the biggest semiprincipal axle length of the generated ellipsoids, we can set $d=d^{\prime}-a$, which is smaller than the actual distance. This approximation actually confines the ellipsoids into those around $O\left(x_{0}, y_{0}, z_{0}\right)$ and makes the computation more efficient.

\section{Application in the Numerical Simulation of Concretes}

4.1. Simulation of a Concrete Specimen Where Aggregates Are Set to Be Ellipsoids. Concrete is a composite threephase material of inhomogeneities in mesoscopic level. These inhonogeneities include coarse aggregate, cement mortar, and the interfacial zones between them. When the aggregates are pepplestones, we can assume them to be ellipsoids. Therefore, the concrete specimen to be simulated is approximately presented as a cuboid with a lot of randomly distributed ellipsoids. All the numerical results are implemented with Matlab on a computer with AMD Phenom(tm) 9500 Quad Core CPU 2.20 GHz and 2.0 GB computer memory.

Before we present the numerical results, it is worthwhile to explain two more things which we have in mind. The first one is about the generation of the interfacial zones. To describe the interfacial zones, we use the generated ellipsoids as outer surface of the interface and generate another ellipsoid with the semiprincipal axes lengths which are $0.003 \mathrm{~cm}$ smaller than the outer ellipsoid, as surface of the aggregate. An example is shown in Figure 2. The second one is about the order of the generation of ellipsoids. According to the grading, we generate all the ellipsoids in order of grading. For example, for the three-grading concretes, we first generate all the ellipsoids of the first grade, then turn to all the ellipsoids of the second grade, and finally generate the ellipsoids of the third grade. The unit of length of all the numerical results is centimeter.

Figure 3 is a simulation of a specimen of two-graded with pebble aggregate concrete where the interfacial zones are also generated. The region size is $[0,15] \times[0,15] \times[0,15]$. All the random variables are assumed to satisfy some uniform distribution. The diameters of the aggregate grains are in two levels, which are 2 4 and 0.5 2, respectively. The aggregate numbers of these two kinds are 107 and 4245, respectively, and the volumes of these two kinds aggregate are 836.2310 and 683.4728 , respectively. The volume ratio of them is about $5.5: 4.5$. The aggregate total volume is 1519.7038 , which is about $45.03 \%$ of the total volume of the specimen. If the bulk density of the aggregates is taken as $27 \mathrm{kN} / \mathrm{m}^{3}$ and the bulk density of the interface is taken as $21 \mathrm{kN} / \mathrm{m}^{3}$, then the weight ratio of aggregates is 0.5129 . The computational time is about 216 hours.

In Figure 4, we present a simulation of a specimen of three-graded with pebble aggregate concrete where cement mortar and the interfacial zones are not included. The region size is $[0,30] \times[0,30] \times[0,30]$. All the random variables are assumed to satisfy some uniform distribution. The diameters of the aggregate grains are in three levels, which are $4 \sim$ $8,2 \sim 4$, and $0.5 \sim 2$, respectively. The aggregate numbers of these three kinds are 34,379, and 26198, respectively, and the

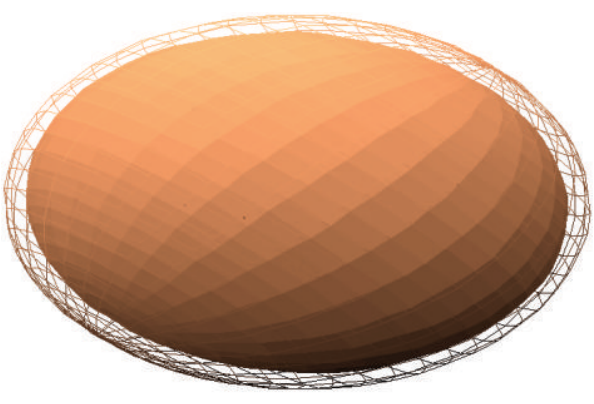

FIGURE 2: The aggregate and interface.

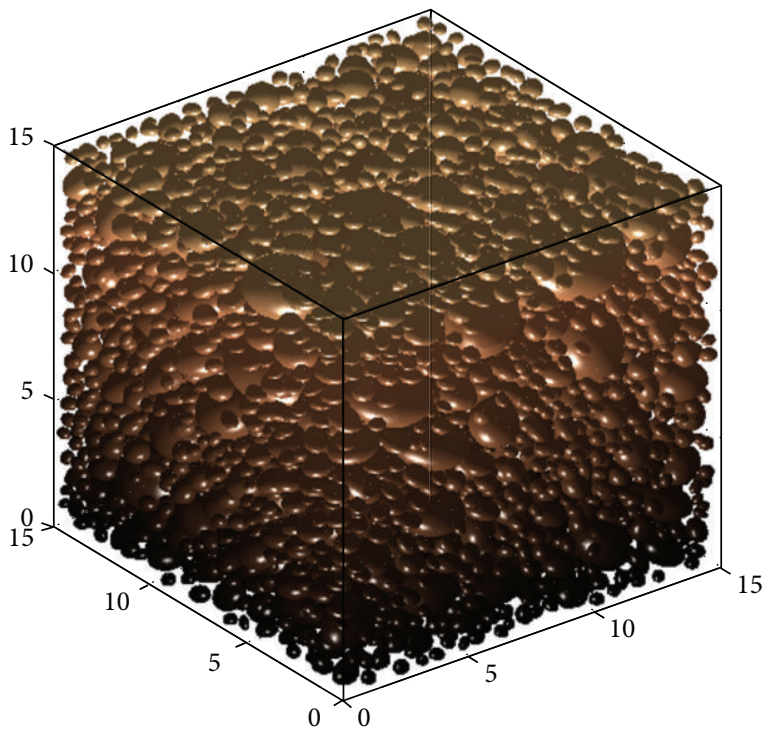

Figure 3: Two-graded ellipsoids randomly distributed region, where the aggregate volume ratio is higher than $45.30 \%$.

volumes of these three aggregates are 5489.1000, 4050.0000, and 4050.0000 , respectively. The volume ratio is about $4: 3: 3$. The aggregate total volume is 13589.0000 , about $50.33 \%$ of the total volume of the specimen. The computational time is about 389 hours.

In Figure 5, we present a simulation of a specimen of fourgraded with pebble aggregate concrete where cement mortar and the interfacial zones are not included. The region size is $[0,45] \times[0,45] \times[0,45]$. All the random variables are assumed to satisfy some uniform distribution. The diameters of the aggregate grains are in four levels, which are $8 \sim 15,4 \sim 8$, $2 \sim 4$, and $0.5 \sim 2$, respectively. The aggregate numbers of these four kinds are 19, 178, 1079, and 53704, respectively, and the volumes of these four aggregates are 15035.6250, 15081.1875, 10023.7500 , and 10023.7500, respectively. The volume ratio is about $3: 3: 2: 2$. The aggregate total volume is 50164.31250 , about $55.05 \%$ of the total volume of the specimen. The computational time is about 227 hours.

In the end of this subsection, we will present another numerical result which is an extension of the numerical results shown above. In this example, we use the contortion of ellipsoids instead of ellipsoids themselves to simulate the 


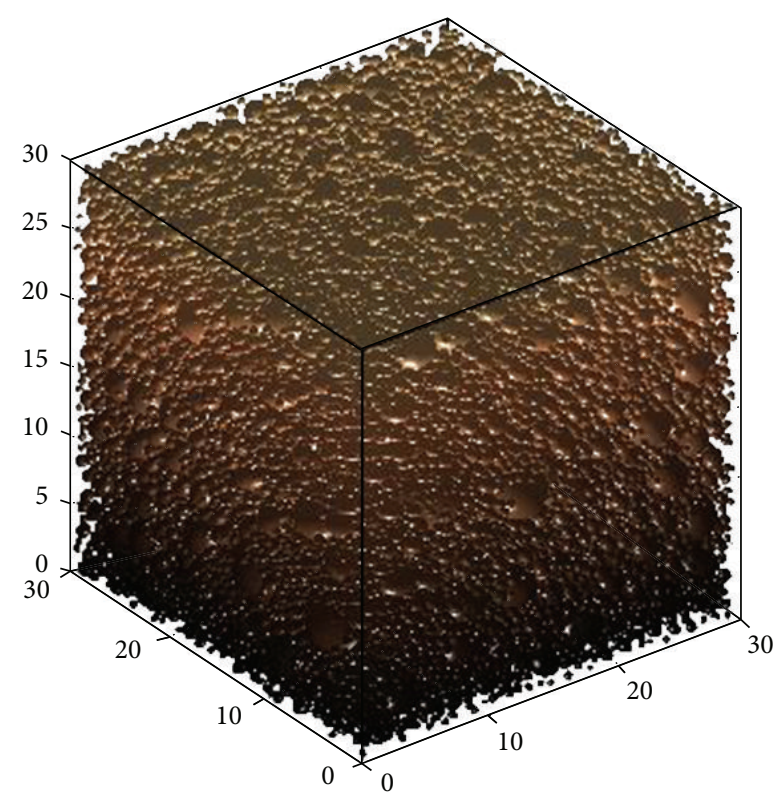

FIGURE 4: Three-graded region with randomly distributed ellipsoids, where the aggregate volume ratio is higher than $50 \%$.

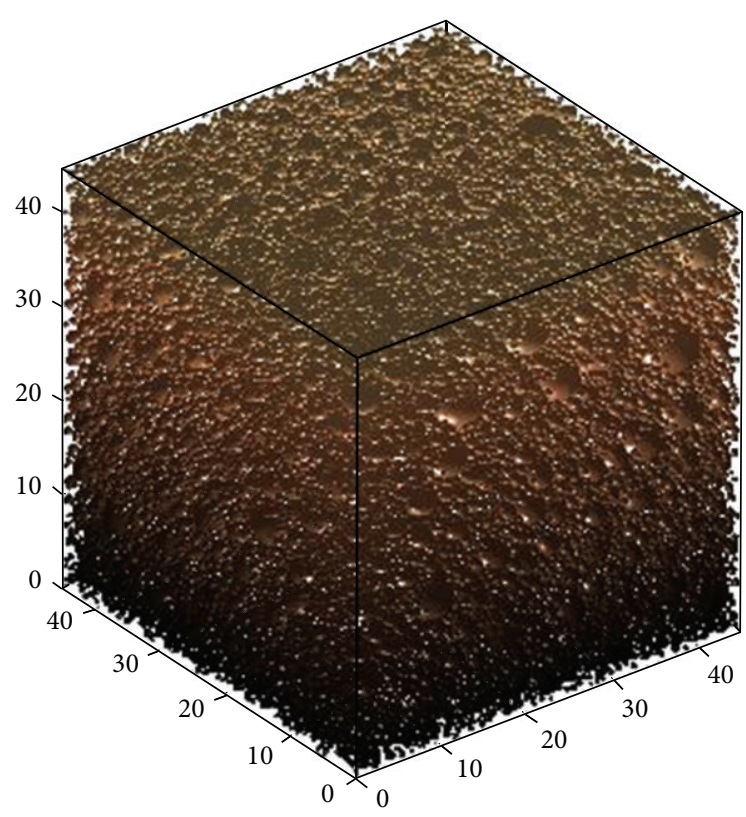

FIGURE 5: Four-graded region with randomly distributed ellipsoids, where the aggregate volume ratio is higher than $55 \%$.

cobble aggregates. The contortion of ellipsoids is based on the free-form deformation method, where the contractionexpansion factor is used to control the deforming of an ellipsoid which is expressed by parametric equation. For more information of the deformation of ellipsoid, please refer to [14]. In Figure 6, we give a model of the deformation of ellipsoid and corresponding interface. In Figure 7, we present an example which is based on Figure 3. All the ellipsoids and interfaces in Figure 3 are deformed to generate Figure 8. In

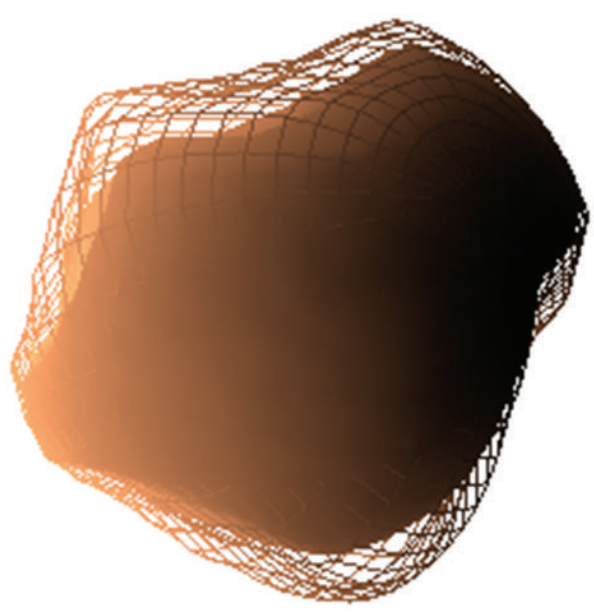

Figure 6: Cobble particles and interface.

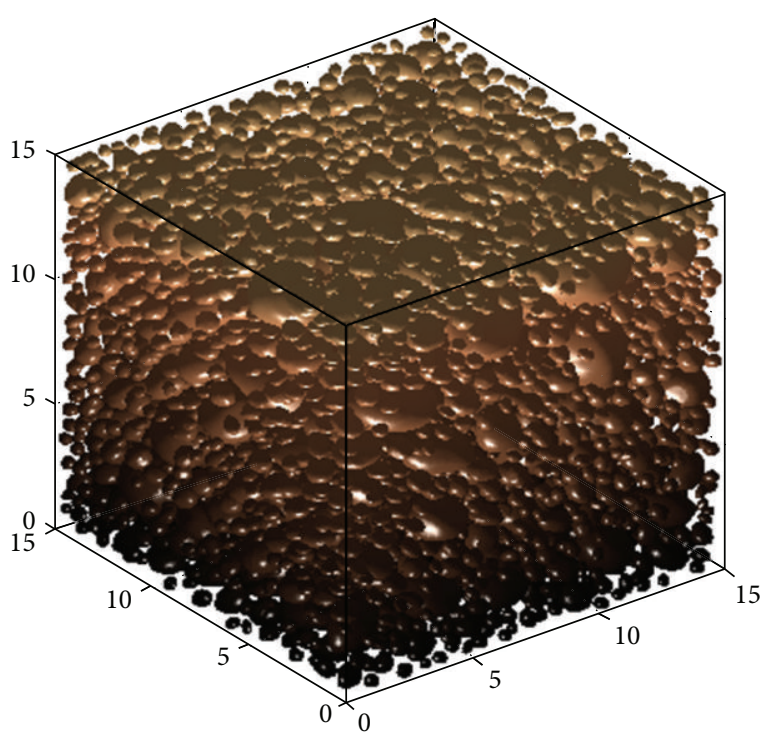

FIgURE 7: Two-graded irregular cobble particles random distribution region, where the aggregate volume ratio is higher than $43.2 \%$.

this case, the volume of all aggregates is 1458.1003 , which is about $43.20 \%$ of the whole volume. The computational time is about 10.5 hours.

4.2. The Finite Element Mesh Generation and Stress Analysis. To demonstrate the effectiveness of the proposed method in the research of the concrete numerical simulation, we are going to do a mesomechanics analysis of concrete with a nonlinear finite element stress analysis on the specimen shown in Figure 4. The corresponding finite element mesh is generated by ABAQUS software, where we treat the concrete specimen as mixed two-phase materials and ignore the interface units. In the ABAQUS, the background grid method is used to determine different content with different phase and all the background grid is the type of C3D8R unit. The unit edge length in the mesh is set as $3 \mathrm{~mm}$. The final finite 


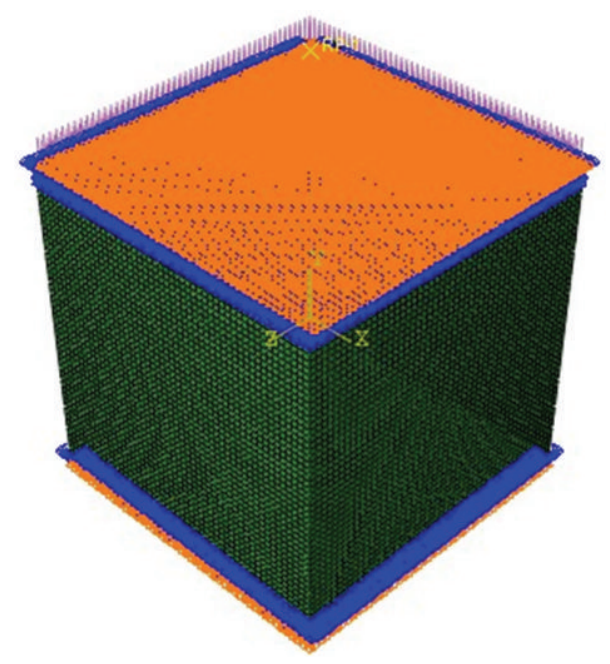

Figure 8: Constraints establishment and load displacement.

TABLE 1: The mechanical parameters of different phase materials.

\begin{tabular}{lcccc}
\hline $\begin{array}{l}\text { Material } \\
\text { type }\end{array}$ & $\begin{array}{c}\text { Elastic } \\
\text { modulus } \\
(/ \mathrm{Gpa})\end{array}$ & $\begin{array}{c}\text { Poisson } \\
\text { ratio }\end{array}$ & $\begin{array}{c}\text { Tensile } \\
\text { strength } \\
(/ \mathrm{Mpa})\end{array}$ & $\begin{array}{c}\text { Ultimate } \\
\text { cracking } \\
\text { strain } \\
(\mathrm{mm} / \mathrm{m})\end{array}$ \\
\hline Mortar & 26 & 0.22 & 3.0 & 0.5 \\
\hline Aggregates & 55.5 & 0.16 & - & - \\
\hline
\end{tabular}

element mesh consists of 132651 knots, 125000 background units, 48079 aggregate units, and 76921 mortar units.

To see whether the finite elements mesh generated above is effective or not, we do the uniaxial tensile test. Specifically, the test is to make the vertical displacement with constraints in the bottom of the specimen, where we fix the central point of the bottom. To get the softening curve, we use the displacement loading method, where $0.1 \mathrm{~mm}$ deformation is loaded from the top of the specimen and the displacement increment is controlled. The mechanical parameters are listed in Table 1. The constraints establishment and the load displacement are illustrated in Figure 8. Finally, we present the nephogram of the damage of the specimen in Figure 9 as well as the tensile damage image of the specimen units in Figure 10.

From the damage plan of the concrete specimen we can see that most of the damaged parts happened in the central part of the specimen. The cracks first occur in the mortar units and then keep extending to pass through the specimen until the specimen is destroyed. During this process, the cracks are always close to the aggregates but never pass through the aggregates. These phenomena agree with the real-world static loading and demonstrate that the specimen generated by the proposed method can be used to further research in the field of cement concrete.

\section{Conclusions}

In this paper we propose one novel method to simulate a domain with large numbers of randomly distributed

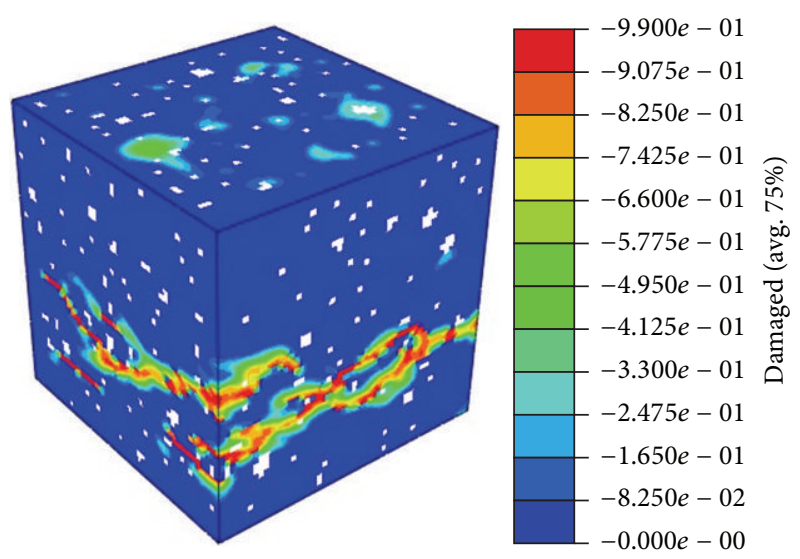

Figure 9: Nephogram of the damaged.
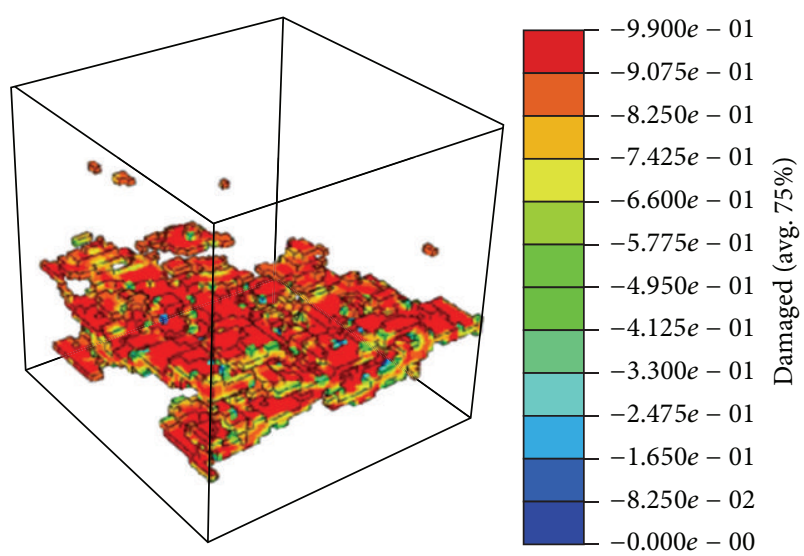

FIgURE 10: The tensile damage image.

ellipsoids. Numerical results show that the proposed method is effective and efficient. Comparing with the existing methods, the proposed method has the following advantages. (1) In the simulation of the concrete material, the proposed method can generate a specimen with ellipsoids aggregates content higher than 55\% according to four-graded aggregates, higher than $50 \%$ according to three-graded aggregates, and higher than $45 \%$ according to two-graded aggregates, respectively. Considering that it is hard for the existing methods to generate a specimen with aggregate content higher than $30 \%$, the proposed method gives a great improvement. (2) The proposed method makes the best of the parametric equation of ellipsoid to determine the relation between a spatial point and the generated ellipsoids and to calculate the distance between a spatial point and the generated ellipsoids. This idea reduces not only the computational complexity in a great degree but also the repellence between different ellipsoids. (3) If the ellipsoids generated by the proposed method are used as covering interface then the cobble aggregates and crushed stone aggregates can be easily achieved with proper deformation. These deformations, actually, can present more geometric models of the concrete aggregates, which can be used in the mesolevel mechanical analysis of the concretes. 


\section{Conflict of Interests}

The authors declare that there is no conflict of interests regarding the publication of this paper.

\section{Acknowledgments}

The work described in this paper is supported by the Natural Science Foundation of China (Project no. 11171181). The last author is partially supported by China Scholarship Council.

\section{References}

[1] Y. B. Zaitsev and F. H. Wittmann, "Simulation of crack propagation and failure of concrete," Matériaux et Construction, vol. 14, no. 5, pp. 357-365, 1981.

[2] L. L. Mishnaevsky Jr., "Three-dimensional numerical testing of microstructures of particle reinforced composites," Acta Materialia, vol. 52, no. 14, pp. 4177-4188, 2004.

[3] J. Segurado, C. González, and J. LLorca, "A numerical investigation of the effect of particle clustering on the mechanical properties of composites," Acta Materialia, vol. 51, no. 8, pp. 2355-2369, 2003.

[4] T. M. Müller and F. Raether, "3D modelling of ceramic composites and simulation of their electrical, thermal and elastic properties," Computational Materials Science, vol. 81, pp. 205211, 2014.

[5] Z. M. Wang, A. K. H. Kwan, and H. C. Chan, "Mesoscopic study of concrete I: generation of random aggregate structure and finite element mesh," Computers and Structures, vol. 70, no. 5, pp. 533-544, 1999.

[6] A. K. H. Kwan, Z. M. Wang, and H. C. Chan, "Mesoscopic study of concrete II: nonlinear finite element analysis," Computers and Structures, vol. 70, no. 5, pp. 545-556, 1999.

[7] E. J. Garboczi, “Three-dimensional mathematical analysis of particle shape using X-ray tomography and spherical harmonics: application to aggregates used in concrete," Cement and Concrete Research, vol. 32, no. 10, pp. 1621-1638, 2002.

[8] W. Wang, J. Wang, and M.-S. Kim, "An algebraic condition for the separation of two ellipsoids," Computer Aided Geometric Design, vol. 18, no. 6, pp. 531-539, 2001.

[9] J. P. B. Leite, V. Slowik, and H. Mihashi, "Computer simulation of fracture processes of concrete using mesolevel models of lattice structures," Cement and Concrete Research, vol. 34, no. 6, pp. 1025-1033, 2004.

[10] S. Häfner, S. Eckardt, T. Luther, and C. Könke, "Mesoscale modeling of concrete: geometry and numerics," Computers and Structures, vol. 84, no. 7, pp. 450-461, 2006.

[11] Y. Yu, J. Cui, and F. Han, "An effective computer generation method for the composites with random distribution of large numbers of heterogeneous grains," Composites Science and Technology, vol. 68, no. 12, pp. 2543-2550, 2008.

[12] J. F. Unger and S. Eckardt, "Multiscale modeling of concrete: from mesoscale to macroscale," Archives of Computational Methods in Engineering, vol. 18, no. 3, pp. 341-393, 2011.

[13] L. Z. Song and B. W. Song, "Approximations to a quadratic programming with quadratic constraints and their applications," Journal of Information and Computational Science, vol. 9, no. 11, pp. 3045-3054, 2012.

[14] L. Z. Song, G. Peng, and W. Y. Yang, "Extension factor for surfaces with maximum in region experiments," Computer
Engineering and Applications, vol. 45, no. 7, pp. 187-189, 2009 (Chinese). 


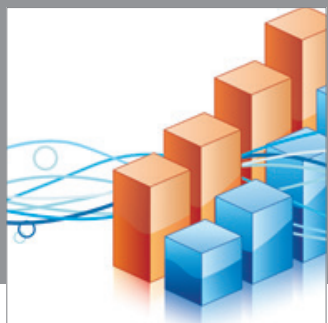

Advances in

Operations Research

mansans

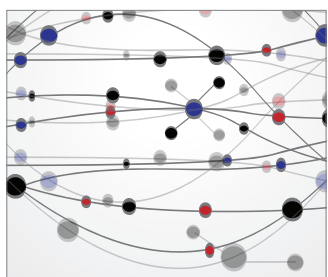

The Scientific World Journal
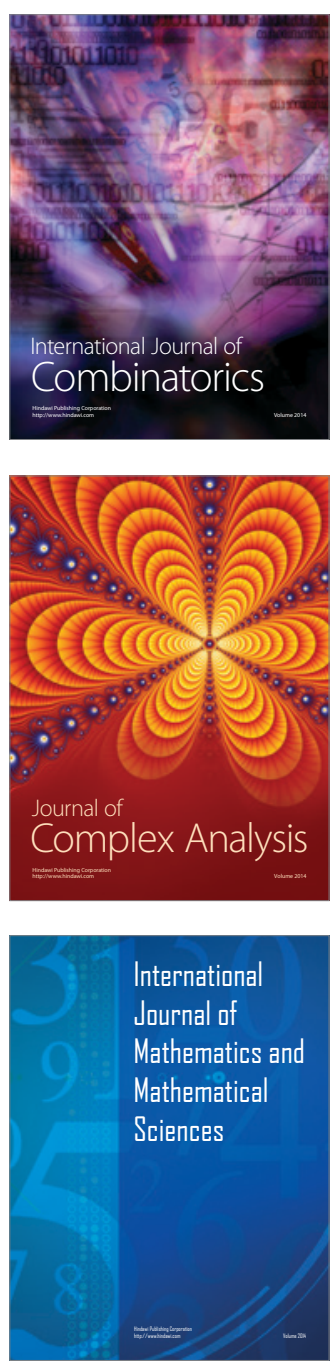
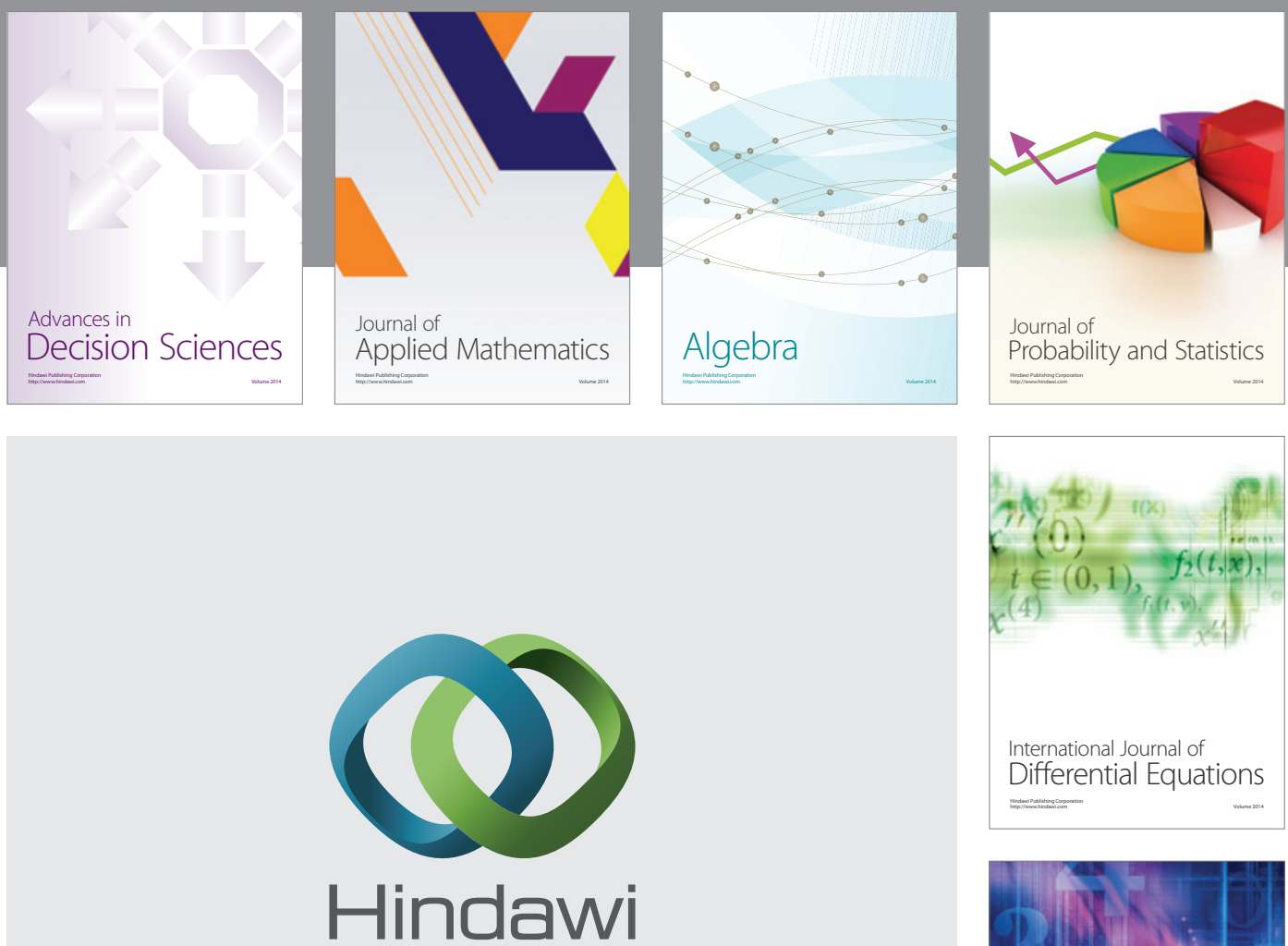

Submit your manuscripts at http://www.hindawi.com
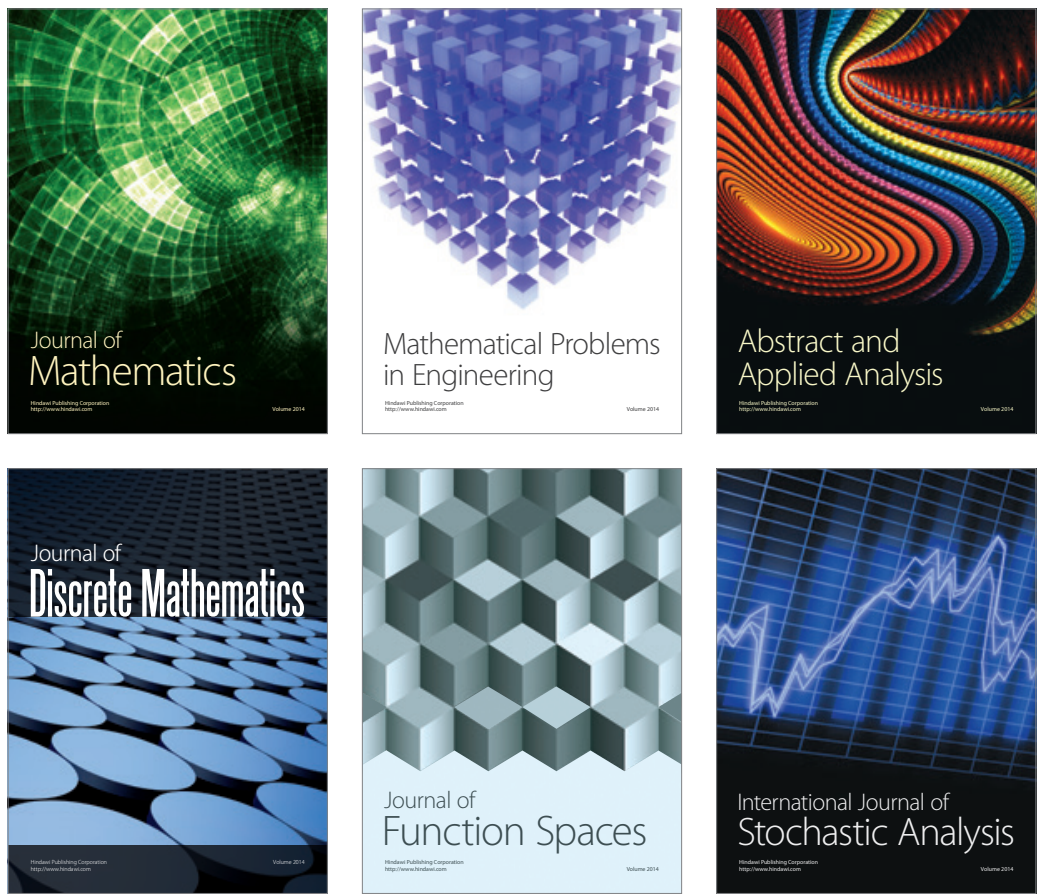

Journal of

Function Spaces

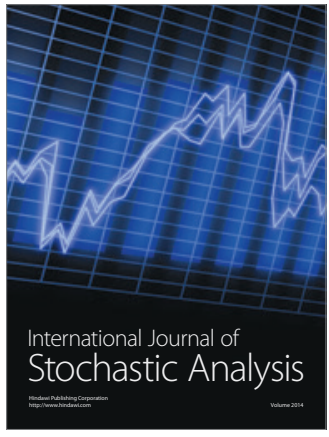

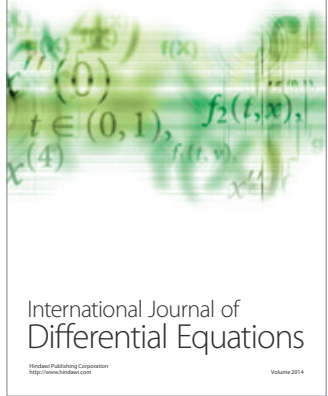
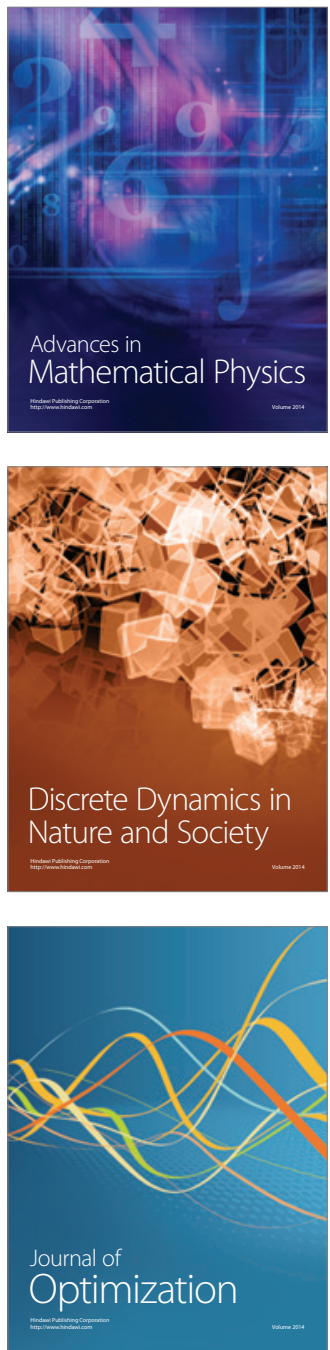